\title{
The evidence for the evolution of interplanetary small flux ropes: Boundary layers
}

\author{
FENG HengQiang ${ }^{1,2,3^{*}}$, WANG JieMin ${ }^{1} \&$ WU DeJin ${ }^{4}$ \\ ${ }^{1}$ College of Physics and Electronic Information, Luoyang Normal University, Luoyang 471022, China; \\ ${ }^{2}$ State Key Laboratory of Space Weather, Center for Space Science and Applied Research, Chinese Academy of Sciences, Beijing 100190, China; \\ ${ }^{3}$ Key Laboratory of Solar Activity, National Astronomical Observatories, Chinese Academy of Sciences, Beijing 100012, China; \\ ${ }^{4}$ Purple Mountain Observatory, Chinese Academy of Sciences, Nanjing 210008, China
}

Received September 6, 2011; accepted November 18, 2011; published online February 21, 2012

\begin{abstract}
We have examined the Wind data in 1996 and identified 21 small interplanetary magnetic flux ropes (SIMFRs), and all the 21 SIMFRs have boundary layer structures. The durations of the boundary layers varied from several minutes to 30 minutes. These boundary layers also have properties of high proton temperature, density, and plasma beta. These boundary layers are formed by magnetic reconnections. In addition, in three events magnetic reconnections were occurring inside the boundary layers. It indicates that the flux rope structures have propagated for some period of time, and their boundaries were still evolving through interaction with the background solar wind. Namely it is very possible that the SIMFRs came from the solar corona.
\end{abstract}

interplanetary small flux ropes, boundary layer, magnetic reconnection exhaust

Citation: Feng H Q, Wang J M, Wu D J. The evidence for the evolution of interplanetary small flux ropes: Boundary layers. Chin Sci Bull, 2012, 57: 1415-1420, doi: 10.1007/s11434-011-4960-7

The interplanetary magnetic flux ropes (IMFRs) are commonly observed by spacecraft in interplanetary space near $1 \mathrm{AU}$, their diameter distribution is in the range of 0.0039 0.6266 AU [1-3]. Among these IMFRs, magnetic clouds (MCs) have large-scale structures whose diameters are more than 0.20 AU [4-6]. MCs are an important subset of interplanetary coronal mass ejections (ICMEs), which are interplanetary manifestations of transient events with large amounts of material ejected from the solar atmosphere. A MC was originally defined empirically in terms of in situ spacecraft measurements of magnetic fields and particles in the interplanetary medium at $\sim 1 \mathrm{AU}$, viz., it has the following necessary properties: (1) the magnetic field direction rotates smoothly through a large angle during an interval of the order of 1 day; (2) the magnetic field strength is higher than average; and (3) low proton temperature compared to the ambient proton temperature [7]. MCs have received considerable attention and have been intensively investigated by many

*Corresponding author (email: fenghq9921@ 163.com) people in the last decade with a focus on the magnetic and plasma structures, its solar origins, and relevant geomagnetic activities [8-15]. In addition to large-scale MCs, only a few studies have been conducted on small interplanetary magnetic flux ropes (SIMFRs), and their origin still remains disputed [2,3,16-18]. Moldwin et al. first reported several SIMFRs, and suggested that these SIMFRs may be a result of the magnetic reconnection at the heliospheric current sheet at $\sim 1$ AU [1,2]. Their main evidence is the lack of intermediatesized events (durations of several hours). If SIMFRs and MCs are of the same physical origin, the size distribution should be continuous. Then Wei et al. suggested that SIMFRs had a larger scale initially and came from the corona, and their magnetic fields were peeled off by the magnetic reconnections near their boundaries when moving from the Sun to the Earth and at last they reached a small scale [19]. Recently, Feng et al. found that IMFRs have a continuous size distribution. Their analysis of IMFRs with different scales showed that the physical properties of the IMFRs changed slowly with their increasing scales [3]. Therefore, Feng et al. 
suggested that SIMFRs also originate in the solar corona and are interplanetary manifestations of small solar transients.

The identification of boundaries of IMFRs has been an important problem in the investigations related to IMFRs, however, the boundaries of magnetic flux ropes are not always evident. Many properties of MCs have been found and are used to confirm the MC boundaries, such as the low plasma beta [20], the presence of increased helium abundance relative to protons [21], bi-directional ion and electron beams [22,23], an increase in the magnetic field strength, the smooth rotation of magnetic field direction, the low proton and electron temperature [24]. However, different criteria may yield different boundaries, and some approaches are to no avail in identifying the boundary of a certain event. Thus these ambiguities make the identification of MC boundaries a difficult problem. Wei et al. found that the magnetic cloud boundary is not a simple discontinuity based on the statistical analysis of many magnetic clouds [19]. They found that the magnetic cloud boundary is a boundary layer that is formed through the interactions between the magnetic cloud and the interplanetary medium (namely magnetic reconnection). Most MCs have boundary layers displaying a drop in magnetic field magnitude and a significant change in field direction. These boundary layers also have properties of high proton temperature, density, and plasma beta. Wei et al. argued that these boundary layers can form through the magnetic reconnection process as a result of the interactions between the MCs and the ambient medium, and the dimensions of IMFRs can decrease due to the magnetic reconnections near their boundaries as they propagate away from the Sun. Then, Wei et al. suggested that small IMFRs may have a larger scale initially, but when the magnetic reconnection process occurs near the boundaries, their magnetic fields are peeled off when moving from the Sun to the Earth and at last they reached a small scale [19]. If their suggestion is correct, these small IMFRs should also have the boundary layer structure. In addition, if SIMFRs came from the corona, the SIMFRs should also have the boundary layer structure. Our purpose is to investigate whether SIMFRs have the boundary layer structures or not The boundary layers were formed through the interactions between the IMFRs and the interplanetary medium when the IMFRs propagate in interplanetary space. Therefore, if almost all the SIMFRs have the boundary layer structures, it means that the flux rope structures have propagated for some period of time, and it is very possible that the SIMFRs came from the solar corona.

\section{Data analysis}

In this study 3-sec-averaged plasma and magnetic field data measured by Wind are used, and the magnetic field data obtained from Magnetic Field Investigation (MFI) magnetometer and the proton data obtained from the 3-Dimension Plasma (3DP) analyzer $[25,26]$. We have examined the Wind data in 1996 and identified 21 SIMFRs which are listed in Table 1. Here, we identify a SIMFR through the following steps: (1) the candidate of SIMFRs is selected by identifying the rotation of magnetic field direction and enhanced magnetic field strength (compared to the ambient medium) with naked eyes; (2) then we examine the correlation coefficient of the velocity and magnetic field components to exclude the possible Alfvén wave structures, if their field and velocity components have a correlation coefficient greater than 0.6 , it is considered to be Alfvén wave structures [27-30]; (3) last we use the geometric parameter of the flux rope to identify the possible events, namely we define it as the magnetic field variation which can be fitted to the cylindrical constant-alpha force-free field. When the difference between the model geometry and the observation data is larger than a criterion value $\left(\sqrt{\chi^{2}}=0.3\right)$, the case is not considered as a flux rope. The deviation can be expressed by the minimum chi-square $\chi^{2}$ (see eq. (2) of Feng et al. [17]). A detailed description of the fitting method can be found in Feng et al. [17]. In addition, we also referred to the SIMFR lists published in the literature $[16,17]$.

\section{Results}

According to the boundary layer criteria used by Wei et al. [19], the high-resolution (3 s) magnetic field and plasma data from Wind were examined; it was found that all the 21 SIMFRs have boundary layer structures. Table 1 lists the identi-

Table 1 The boundary layers of Small interplanetary flux ropes in 1996

\begin{tabular}{ccccc}
\hline No. $^{\text {a) }}$ & $\mathrm{F}_{1}^{\mathrm{b})}$ & $\mathrm{F}_{2}^{\mathrm{c}}$ & $\mathrm{R}_{1}^{\mathrm{d})}$ & $\mathrm{R}_{2}^{\mathrm{e})}$ \\
\hline 1 & $1996-01-2822: 13$ & $22: 22$ & $22: 58$ & $23: 09$ \\
2 & $1996-02-0302: 38$ & $02: 38$ & $03: 51$ & $04: 07$ \\
3 & $1996-02-0619: 46$ & $20: 01$ & $01: 36 \mathrm{~N}$ & $01: 38 \mathrm{~N}$ \\
4 & $1996-02-0804: 04$ & $04: 30$ & $05: 39$ & $05: 45$ \\
5 & $1996-02-1017: 39$ & $18: 41$ & $22: 08$ & $22: 30$ \\
6 & $1996-02-2404: 38$ & $04: 38$ & $05: 52$ & $06: 07$ \\
7 & $1996-03-0201: 43$ & $01: 58$ & $10: 07$ & $10: 07$ \\
8 & $1996-03-0313: 38$ & $13: 38$ & $14: 55$ & $15: 16$ \\
9 & $1996-03-0723: 58$ & $00: 12 \mathrm{~N}$ & $05: 19 \mathrm{~N}$ & $05: 37 \mathrm{~N}$ \\
10 & $1996-03-0819: 47$ & $19: 47$ & $02: 56 \mathrm{~N}$ & $03: 24 \mathrm{~N}$ \\
11 & $1996-03-0908: 50$ & $09: 19$ & $13: 58$ & $14: 23$ \\
12 & $1996-03-1309: 17$ & $09: 27$ & $10: 21$ & $10: 21$ \\
13 & $1996-04-1514: 31$ & $14: 31$ & $15: 23$ & $15: 33$ \\
14 & $1996-05-0205: 30$ & $05: 44$ & $06: 47$ & $06: 47$ \\
15 & $1996-05-1421: 52$ & $21: 59$ & $23: 09$ & $23: 24$ \\
16 & $1996-05-1700: 42$ & $00: 58$ & $04: 20$ & $04: 50$ \\
17 & $1996-06-2016: 40$ & $16: 47$ & $18: 30$ & $18: 57$ \\
18 & $1996-08-0600: 04$ & $00: 26$ & $03: 35$ & $03: 46$ \\
19 & $1996-08-2901: 10$ & $01: 20$ & $02: 13$ & $02: 13$ \\
20 & $1996-09-0123: 35$ & $23: 37$ & $03: 07 \mathrm{~N}$ & $03: 15 \mathrm{~N}$ \\
21 & $1996-09-1317: 47$ & $17: 51$ & $19: 04$ & $19: 09$ \\
\hline
\end{tabular}

a) The code number of the interplanetary magnetic flux rope; b) the beginning of the front boundary layer (UT); c) the end of the front boundary layer (UT), and $\mathrm{N}$ indicates the time on a following day; d) the beginning of the rear boundary layer (UT); e) the end of the rear boundary layer (UT). 


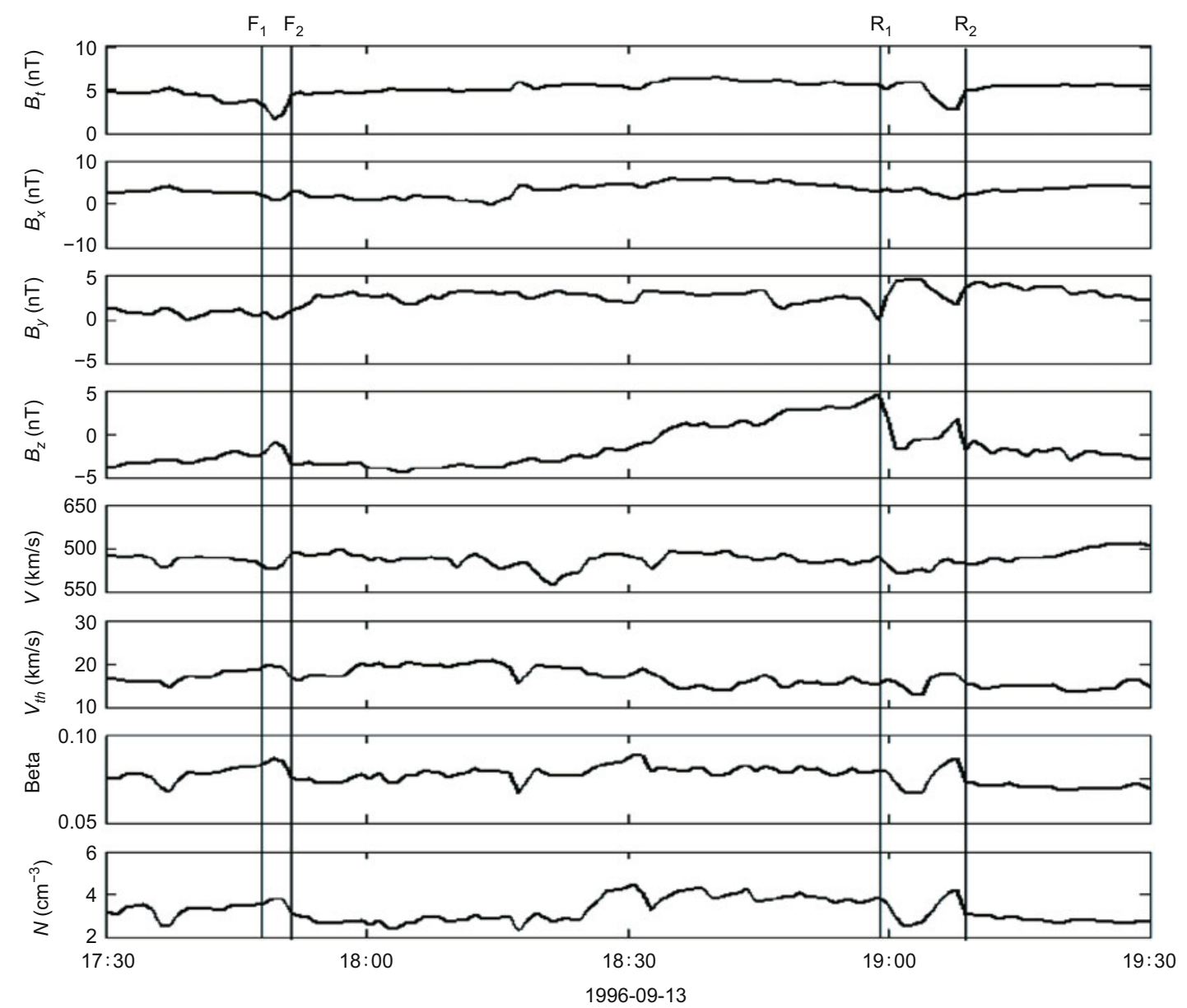

Figure 1 Interplanetary magnetic field and speed data measured by the Wind spacecraft during the 1996 September 13 SIMFR passage. The vertical lines $\mathrm{F}_{1}$ and $F_{2}$ indicate the start and end times of the front boundary layer, and the vertical lines $R_{1}$ and $R_{2}$ indicate the start and end times of the rear boundary layer.

fied boundary layers related to SIMFRs. The second and third columns show the start and end times of the front boundary layer, and the fourth and fifth columns list the start and end times of the rear boundary layer. The durations of the front boundary layers varied from 2 to 29 min with a mean value of 12.5 minutes; the durations of the rear boundary layers varied from 2 to $30 \mathrm{~min}$ with a mean value of $17.1 \mathrm{~min}$. All the boundary layers have the reconnection features, such as the relatively high proton temperature, high proton density, and high plasma beta, a significant change of the observed field orientation. As Wei et al. pointed out, these features are formed by magnetic reconnection. As an example, Figure 1 shows the magnetic field and plasma data of the magnetic reconnection of the 1996 Sep. 13 SIMFR. The magnitude of the total magnetic field $\left(B_{t}\right)$; the $x, y, z$ components of the magnetic field $\left(B_{x}, B_{y}, B_{z}\right)$; the proton speed $(V)$; the proton temperature $\left(T_{P}\right)$; the plasma beta; and the proton density $(N)$ are shown from the top to the bottom of the panels. The coordinate system is the geocentric solar ecliptic ( $G S E)$ Cartesian system. From Figure 1 it is easy to see that the SIMFR displays a smooth rotation of the magnetic field direction. One can also find that the bipolar field appears in the $\left(B_{z}\right)$ component. These are all classic flux rope signatures. The front and rear boundary layers are labeled by "F1-F2" and "R1-R2", respectively. Inside the boundary layers, the total magnetic field strength decreased sharply, reaching a minimum near the center. It can also be seen that the proton temperature, number density, plasma beta, and proton velocity increased considerably compared with the plasma in the adjacent background solar wind and the SIMFR body. They are the typical characteristics of boundary layers. As mentioned above, the relatively high proton temperature, high proton density, and high plasma beta are possibly formed by magnetic reconnection, and the outer boundary is a possible happened reconnection exhaust boundary. Among the 21 SIMFRs, three events provided sure-enough evidence of An example will be presented later magnetic reconnections were happening inside the boundary layers. Then we will give a detailed example. In addition, some SIMFRs have no front, in two locations here or rear boundary layers. We further analyzed the observational characteristics of the SIMFR events without font or rear boundary layers and found that the plasma and magnetic field characteristics are similar before and after the boundary, and the orientation of $\mathrm{B}$ straddled a relative small change $\left(<100^{\circ}\right)$.

Figure 2 shows the velocity and interplanetary field data 


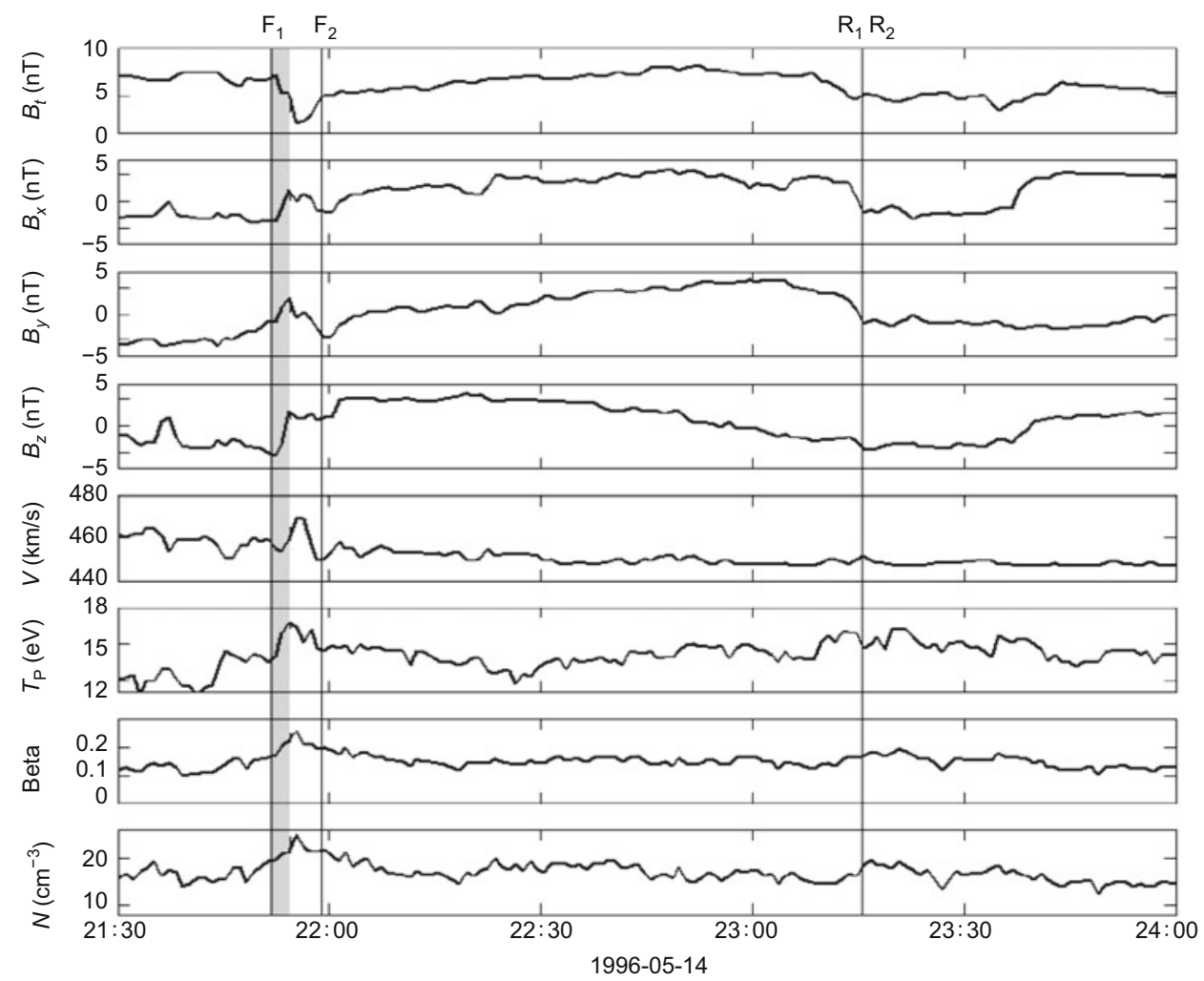

Figure 2 Interplanetary magnetic field and speed data measured by the Wind spacecraft during the 1996 May 14 SIMFR passage. The vertical lines $\mathrm{F}_{1}$ and $F_{2}$ indicate the start and end times of the front boundary layer. The SIMFR has no rear boundary layer, so the $R_{1}$ and $R_{2}$ are at the same time. The gray shaded region indicates the magnetic reconnection exhaust.

on 1996 May 14. From Figure 2 it is easy to see that the SIMFR displays a smooth rotation of the magnetic field direction and enhanced magnetic field magnitude. One can also find that the bipolar field appears in the $B_{z}$ component, and the core field appears in the $B_{x}$ and $B_{y}$ components. From Figure 2, we can find that there is a magnetic reconnection exhaust (the shaded region) inside the front boundary layer, and the leading boundary of the magnetic reconnection exhaust is just the outer boundary of the front boundary layer. In addition, Figure 3 also provides expanded views of the magnetic field and velocity curves of the magnetic reconnection exhaust. As can be seen from Figures 2 and 3, the reconnection exhaust is within 21:53:05-21:53:58 UT; the change of the observed field orientation in this exhaust is large $\left(122^{\circ}\right)$; within the exhaust the plasma beta, the proton temperature and density are all higher than outside; The magnetic field strength is weaker than that of the surrounding solar wind; the changes in $\boldsymbol{V}$ and $\boldsymbol{B}$ are correlated with one another at the front boundary of the event and are anticorrelated at the rear boundary; the ambient solar wind has a low proton beta $(<0.3)$. These features satisfy the criteria of the reconnection exhausts [31]. These observations indicated that the magnetic flux in the flux rope was decreasing by the magnetic reconnection. It means that the magnetic fields are peeling off from the magnetic flux rope. Therefore, the spatial scale of the flux rope would diminish gradually, and their boundaries were still evolving through interaction with the background solar wind, as suggested by Wei et al. [19].

\section{Summary and discussion}

SIMFRs are widely observed and reported at $1 \mathrm{AU}$ in recent years, but their origin is still debatable $[2,3,16,17]$. The current studies indicate the generation of SIMFRs has two possibilities. One is that these SIMFRs result from magnetic reconnection in the solar wind well away from the Sun $[2,16]$. The other one is that the SIMFRs are produced by solar eruptions $[3,17,19]$, that is to say, they are the interplanetary manifestations of small CMEs, or they have a larger scale initially, but when the magnetic reconnection process occurs near the boundaries, their magnetic fields are peeled off when moving from the Sun to the Earth and at last they reach a small scale [19]. If these small IMFRs came from the corona, like MCs, the SIMFRs should also have the boundary layer structures. We have examined the magnetic field and plasma data around the boundary of the SIMFRs in 1996, and found that all the identified 21 SIMFRs have boundary layer structures. Like the duration of SIMFR is shorter than that of MC, the duration of the boundary layer of SIMFR is also shorter. The durations of the boundary layers varied from several minutes to 30 minutes. These boundary layers also have properties of high proton temperature, density, and plasma beta. In addition, three magnetic reconnection exhausts were found inside 


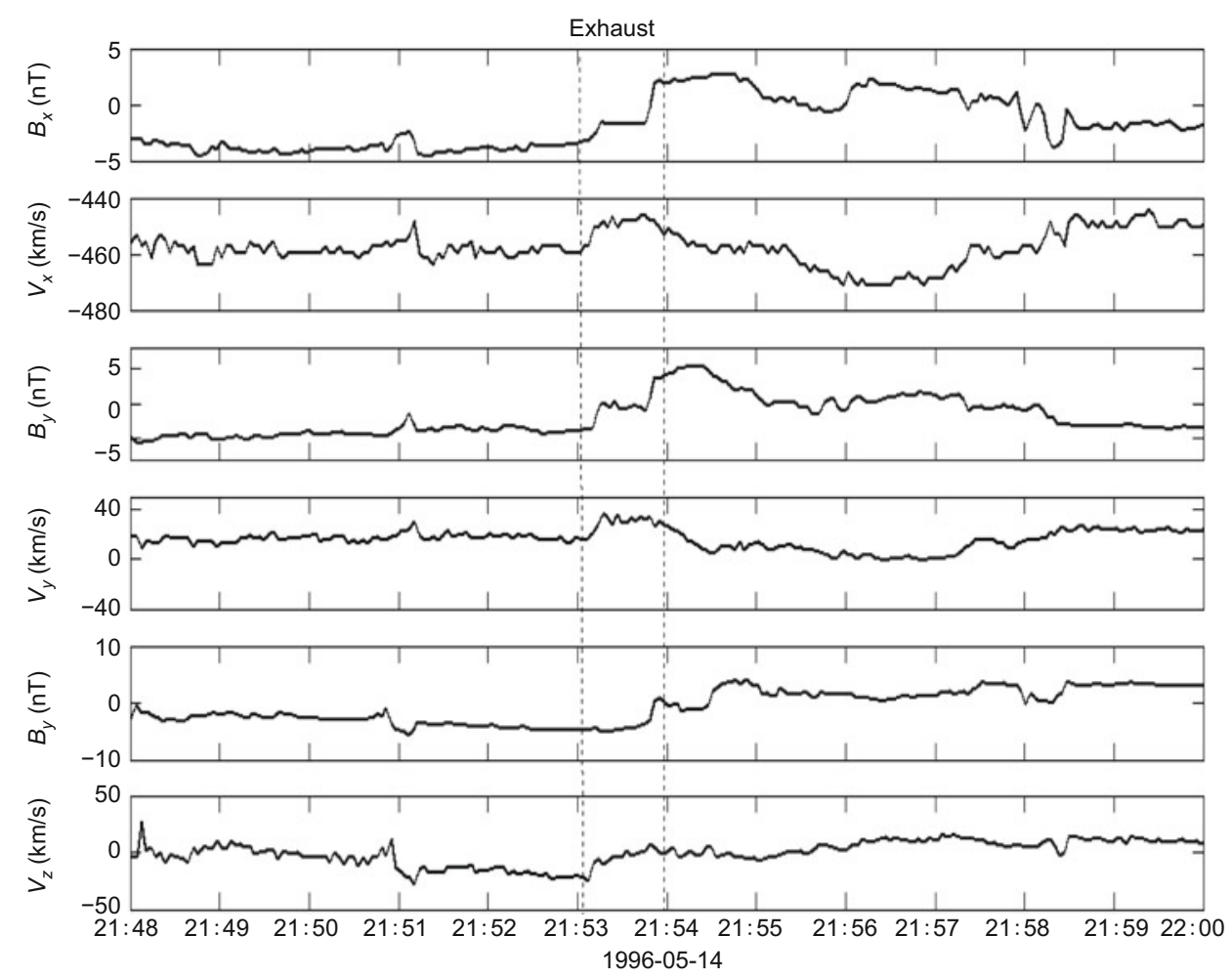

Figure 3 Interplanetary magnetic field and speed data measured by the Wind spacecraft during the 1996 May 14 magnetic reconnection exhaust passage. The dotted vertical lines indicate the beginning and the end of the reconnection exhaust.

the boundary layers. It implies that the boundary layer was formed through the magnetic reconnection process as a result of the interactions between the magnetic cloud and the interplanetary medium. Therefore, it is possible that some SIMFRs have larger scales initially and come from the corona; their magnetic fields are peeled off when moving from the Sun to the Earth, where they reach small scales [19]. Nevertheless, it is also possible that these SIMFRs have less scales than normal MCs when they erupt in the corona.These observational facts at least indicated that the flux rope structures have propagated for some time, and their boundaries were still evolving through interaction with the background solar. Namely it is very possible that the SIMFRs came from the solar corona. In addition, there is a large body of evidence of small eruptions in the literature, such as small CME, plasma blobs, X-ray bright sigmoidal structures, X-ray plasmoids [32-38]. The correlation between the small eruptions and small-scale IMFRs may exist and our future work will include a survey of their correlation.

The authors thank NASA/GSFC for the use of data from Wind. This work was supported by the National Natural Science Foundation of China (41074124, 40804034 and 40890162), the Program for Science \& Technology Innovation Talents in Universities of Henan Province (HASTIT) (2010HASTIT022), the Specialized Research Fund for State Key Laboratories and Key Laboratory of Solar Activity and the Program for Science \& Technology of Henan (092300410189).

1 Moldwin M B, Phillips J L, Gosling J T, et al. Ulysses observation of a noncoronal mass ejection flux rope: Evidence of interplanetary magnetic reconnection. J Geophys Res, 1995, 100: 19903-19910
2 Moldwin M B, Ford S, Lepping R, et al. Small-scale magnetic flux ropes in the solar wind. Geophys Res Lett, 2000, 27: 57-60

3 Feng H Q, Wu D J, Chao J K. Size and energy distributions of interplanetary magnetic flux ropes. J Geophys Res, 2007, 112: A02102

4 Burlaga L F. Magnetic clouds and force-free fields with constant alpha. J Geophys Res, 1988, 93: 7217-7224

5 Lepping R P, Burlaga L F, Jones J A. Magnetic field structure of interplanetary magnetic clouds at 1 AU. J Geophys Res, 1990, 95: $11957-$ 11965

6 Farrugia C J, Osherovich V A, Burlaga L F. Magnetic flux rope versus the spheromak as models for interplanetary magnetic clouds. J Geophys Res, 1995, 100: 12293-12306

7 Burlaga L F, Sittler E, Mariani F, et al. Magnetic loop behind an interplanetary shock: Voyager, Helios, and IMP 8 observations. J Geophys Res, 1981, 86: 6673-6684

8 Bothmer V, Schwenn R. The structure and origin of magnetic clouds in the solar wind. Ann Geophys, 1998, 16: 1-24

9 Webb D F, Cliver E W, Crooker N U, et al. Relationship of halo coronal mass ejections, magnetic clouds, and magnetic storms. J Geophys Res, 2000, 105: 7491-7508

10 Wu D J, Chao J K, Lepping R P. Interaction between an interplanetary magnetic cloud and the Earth's magnetosphere: Motions of the bow shock. J Geophys Res, 2000, 105: 12627-12638

11 Wu C C, Lepping R P, Gopalswamy N. Relationships among magnetic clouds, CMEs and geomagnetic storms. Sol Phys, 2006, 239: 449-460

12 Wang Y, Hong M H, Chen G X, et al. Nightside field-aligned current during the April 6, 2000 superstorm. Chin Sci Bull, 2010, 55: 21752181

13 Yuan C J, Zong Q G. Dynamic variations of the outer radiation belt during magnetic storms for 1.5-6.0 MeV electrons. Sci China Tech Sci, 2011, 54: 431-440

14 Zhang Y, Chen J Y, Feng X S. Predicting the shock arrival time using 1D-HD solar wind model. Chin Sci Bull, 2010, 55: 1053-1058 
15 Lu S, Lu Q M, Cao Y, et al. The effects of the guide field on the structures of electron density depletions in collisionless magnetic reconnection. Chin Sci Bull, 2011, 56: 48-52

16 Cartwright M L, Moldwin M B. Comparison of small-scale flux rope magnetic properties to large-scale magnetic clouds: Evidence for reconnection across the HCS? J Geophys Res, 2008, 113: A09105

17 Feng $\mathrm{H} \mathrm{Q}$, Wu D J, Lin C C, et al. Interplanetary small- and intermediate-sized magnetic flux ropes during 1995-2005. J Geophys Res, 2008, 113: A12105

18 Feng H Q, Wu D J. Observations of a small interplanetary magnetic flux rope associated with a magnetic reconnection. Astrophys J, 2009, 705: 1385-1387

19 Wei F, Liu R, Fan Q, et al. Identification of the magnetic cloud boundary layers. J Geophys Res, 2003, 108: 1263

20 Gosling J T, Baker D N, Bame S G, et al. Bidirectional solar wind electron heat flux events. J Geophys Res, 1987, 92: 8519-8535

21 Zwickl R D, Asbridge J R, Bame S J, et al. Plasma properties of driver gas following interplanetary shocks observed by ISWW-3, in Solar Wind Five. NASA Conf Publ, 1983, 2280: 711-717

22 Marsden R G, Sanderson T R, Tranquiller C, et al. ISEE-3 observations of low energy proton bidirectional events and their relation to isolated interplanetary magnetic structures. J Geophys Res, 1987, 92: 11009_ 11019

23 Gosling J T, Baker D N, Bame S J, et al. Bidirectional solar wind electron heat flux events. J Geophys Res, 1987, 92: 8519-8535

24 Burlaga L F, Skong R M, Smith C W, et al. Fast ejecta during the ascending phase of solar cycle 23: ACE observations, 1998-1999. J Geophys Res, 2001, 106: 20957-20978

25 Lin R P, Anderson K A, Ashford S, et al. A three-dimensional plasma and energetic particle investigation for the wind spacecraft. Space Sci Rev, 1995, 71: 125-153

26 Lepping R P, Acuna M H Burlaga L F, et al. The wind magnetic field investigation. Space Sci Rev, 1995, 71: 207-229

27 Coleman P J. Wave-like phenomena in the interplanetary plasma: Mariner 2. Planet Space Sci, 1967, 15: 953

28 Coleman P J. Turbulence, viscosity, and dissipation in the solar wind plasma. Astrophys J, 1968, 153: 371

29 Belcher J W, Davis Jr L. Large-amplitude Alfvén waves in the interplanetary medium. J Geophys Res, 1971, 76: 3534-3563

30 Burlaga L F, Turner J M. Microscale 'Alfvén waves' in the solar wind at 1 AU. J Geophys Res, 1976, 81: 73-77

31 Gosling J T, Skoug R M, McComas D J, et al. Direct evidence for magnetic reconnection in the solar wind near 1 AU. J Geophys Res, 2005, 110: A01107

32 Chen Y, Li X, Song H Q, et al. Intrinsic instability of coronal ctreamers. Astrophys J, 2009, 191: 1936-1942

33 Sheeley N R, Wang Y M, Hawley S H, et al. Measurements of flow speeds in the corona between 2 and $30 R_{\odot}$. Astrophys J, 1997, 484: $472-478$

34 Song H Q, Kong X L, Chen Y, et al. A Statistical study on the morphology of rays and dynamics of blobs in the Wake of coronal mass ejections. Sol Phys, 2011, doi: 10.1007/s11207-011-9848-9

35 Song H Q, Chen Y, Liu K, et al. Quasi-periodic releases of streamer blobs and velocity variability of the slow solar wind near the Sun. Sol Phys, 2009, 258: 129-140

36 Sterling A C, Hudson H S. YOHKOH SXT observations of X-ray "dimming" associated with a halo coronal mass ejection. Astrophys J, 1997, 491: L55-L58

37 Ohyama M, Shibata K. X-ray plasma ejection associated with an impulsive flare on 1992 October 5: Physical conditions of X-ray plasma ejection. Astrophys J, 1998, 499: 934-944

38 Shibata K, Masuda S, Shimojo M, et al. Hot-plasma ejections associated with compact-loop solar flares. Astrophys J, 1995, 451: L83L85

Open Access This article is distributed under the terms of the Creative Commons Attribution License which permits any use, distribution, and reproduction in any medium, provided the original author(s) and source are credited. 